\title{
A five-stage singly diagonally implicit Runge-Kutta-Nyström method with reduced phase-lag
}

\begin{abstract}
In this paper, a singly diagonally implicit Runge-Kutta-Nyström (RKN) method is constructed for solving second-order ordinary differential equations (ODE) with oscillatory solutions. The produced method is 5-stage, algebraic order five, and phase-lag (or dispersion) order eight at a cost of five function evaluations per step. The method is more accurate compare to current existing similar type of methods for the numerical integration of secondorder differential equations with periodic solutions by using constant step size.
\end{abstract}

Keyword: Diagonally implicit; Oscillatory solutions; Phase-lag; Runge-Kutta-Nyström methods 